\title{
Robot-Assisted First Fib Resection for Neurogenic Thoracic Outlet Syndrome
}

\author{
Peter-J an V, Elke W, Lieven $M$ and Roel B* \\ Department of Cardiovascular Surgery, OLV Hospital, \\ Aalst, Belgium \\ *Corresponding author: Roel Beelen, Department \\ of Cardiovascular and Thoracic Surgery, OLV Hospital, \\ Aalst, Belgium
}

Received: February 06, 2019; Accepted: February 27, 2019; Published: March 06, 2019

\begin{abstract}
Background: When conservative measures fail in the treatment of thoracic outlet syndrome (TOS), a first rib resection (FRR) is in order. Nowadays, a transaxillary or supraclavicular approach is most commonly used. However, both techniques have technical limitations.
\end{abstract}

Objective: In this paper a minimal invasive, robot-assisted thoracoscopic technique tackling those shortcomings is proposed.

Methods: A single-center retrospective study including 11 patients with 12 FRR's from January 2017 until August 2018 was performed. The main focus was the feasibility of this new technique by looking at mean hospital stay, postoperative pain and complication rate. Clinical outcome was evaluated at 6 weeks.

Results: Twelve FRR were performed in 10 women and 1 man. Average operative time was $99 \pm 32$ minutes. One patient needed chest tube replacement for pneumothorax after initial tube removal. One patient was readmitted for pleurisy which was treated conservatively. Max average pain score (VAS; visual analogue pain score) was 5 . Mean hospital stay was 3.4 days. Outcome at 6 weeks' time showed a complete relief of symptoms in 8 cases. Three patients had some residual fatigue complaints. However, these complaints improved over time. In one case there was no real benefit from the procedure.

Conclusion: Robot-assisted thoracoscopic FRR is a safe and feasible method in the treatment of TOS with excellent postoperative results. A robotassisted approach offers the benefit of an excellent exposure of the operative field and gentle handling of tissue. Further research in larger series is needed to compare this promising technique to conventional approaches.

Keywords: First rib resection; Thoracic outlet; Treatment; Robot-assisted

\section{Introduction}

\section{Definition}

The thoracic outlet is defined as the region where the neurovascular structures (roots of the brachial plexus, subclavian vein and artery) originating from the neck take passage underneath the clavicle, between the scalene muscles and above the first rib towards the arm. A thoracic outlet syndrome (TOS) is a collective term for the impingement of these neurovascular structures [1].

Impingement mostly occurs at the scalene triangle. This triangle consists of the anterior and middle scalene muscle. The base of the triangle is formed by the first rib. The nerve roots of the brachial plexus and the subclavian artery take passage through this triangle. The subclavian vein, on the other hand, runs between the anterior scalene muscle and the subclavius muscle.

Depending on which structure is compressed, a difference is made between neurogenic TOS (nTOS) in 95\%, venous TOS (vTOS) in $4 \%$ and arterial TOS (aTOS) in $1 \%$ of all cases [2].

\section{Pathophysiology}

The mechanisms leading to TOS are numerous and complex. They can be divided into two categories. A first one consists of congenital factors such as a cervical rib, fibro muscular bands, a broad first rib and a prolonged cervical transverse process. A second one consists of acquired, extrinsic factors such as repetitive motion and trauma. This may cause hypertrophy and scarring of the scalene muscles. All these mechanisms lead in some extend to the narrowing of the scalene triangle.

The first rib plays a key role in the development of a TOS because it is on this rigid floor the neurovascular bundle effectively gets impinged. This effect is potentiated by the scalene muscles lifting up the first rib, further reducing the area of the scalene triangle.

This pathophysiological mechanism forms the substrate of the surgical treatment of TOS (cf. infra).

\section{Treatment}

Initial treatment of TOS typically starts with life style changes and physical therapy during 6-8 weeks. Painkillers and muscle relaxants may also be prescribed. If this fails, a scalene block (botox/lidocaine) can be proposed, lowering the first rib and thus increasing the surface of the scalene triangle [2]. All of these measures are limited in effect and duration. 
Often, patients need a more profound cure in the form of a first rib resection with consequently release of the scalene muscles. By removing the bony base of the scalene triangle, the neurovascular structures are no longer limited to a narrow space. This results in a significant reduction or complete relieve of symptoms in up to $90 \%$ of the cases [3].

\section{A new approach}

Nowadays, a transaxillary or supraclavicular approach is most commonly used in FRR [2]. A transaxillary approach is limited by the narrow, deep axilla. Furthermore there is a risk of injuring the intercostobrachial nerve. A supraclavicular approach is preferred when arterial reconstruction is necessary. However, the brachial plexus, the phrenic nerve and the long thoracic nerve are more easily damaged by this technique.

A first minimal invasive approach (VATS) has been described as well, but never gained much interest [4-6]. This has probably to do with the current limitations of the video-assisted thoracoscopic instrumentation, making it hard to reach the first rib.

In this paper a robot-assisted thoracoscopic approach, tackling the shortcomings of the techniques described above is proposed. During the writing of this article, this approach has been described in the treatment in vTOS (Paget-Schroetter disease) [7-10] and in the treatment of aTOS caused by a cervical rib [11]. Recently published an article treating 4 cases of nTOSrobotically (April 2018) [12].

\section{Surgical technique}

The goal of the procedure is to remove as much of the first rib as possible $(6 \mathrm{~cm})$ with a release of the costoclavicular ligament, anterior and medial scalene muscle. The costovertebral and costosternaljoints are not resected to avoid first rib instability.

\section{Step 1}

After intubation with a double-lumen endotracheal tube, deflating the lung at the affected side, the patient is placed in lateral decubitus with the effected side facing upwards and the arm positioned above the patients head.

This set-up, together with the use of carbon dioxide insufflation (8 $\mathrm{mmHg}$ ), through anEndopath Xcel trocar (Ethicon, Inc, Bridgewater, NJ, USA), opens up the thoracic outlet as much as possible and helps pulling the lung away from the first rib. An intra-thoracic lung retractor is not used.

The da Vinci Si robot (Intuitive Surgical, Inc, Sunnyvale, CA, USA) is used [13], which is positioned over the head of the patient. In the authors' opinion, the da Vinci Si robot system offers a more convenient alignment with the patient than the da Vinci Xi system (Figure 1).

The operative field is draped with an antimicrobial drape. The thoracic cavity is then entered via the fourth intercostal space by three incisions, respectively over the anterior, middle and posterior axillary line. The middle incision is used as a camera-port ( $30^{\circ}$ stereoscope). A cautery spatula and a Cadière forceps are introduced via the right and the left port respectively (Figure 2).

\section{Step 2: First rib dissection and incision}

After entering the thoracic cavity, the parietal pleura over the

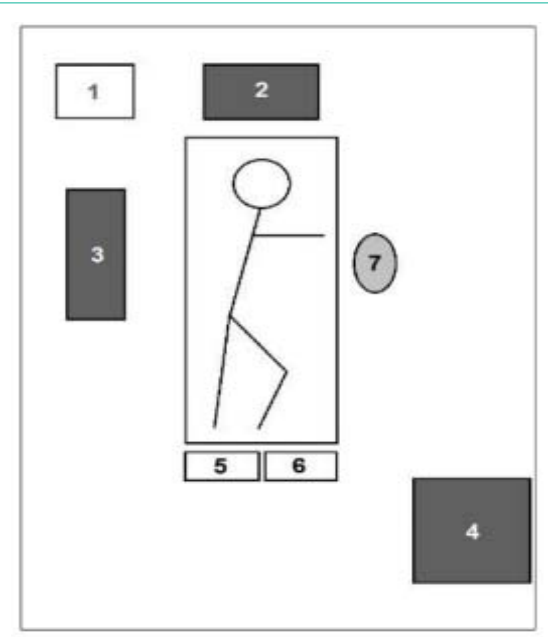

Figure 1: Operating room layout and patient positioning. Numbers 1 to 7 in following order are: anesthesiologist, da Vinci® $\mathrm{Si}$ robot, OR monitor, operative console, Midas Rex® surgical drill, electrosurgical device, scrub nurse. Courtesy of Wybaillie Elke [11].

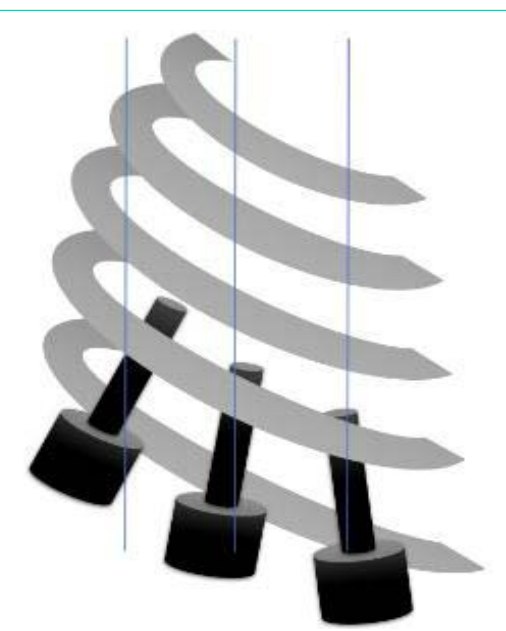

Figure 2: Schematic overview of trocar placement via the fourth intercosta space. From left to right on the posterior, middle and anterior axillary line.

first rib is opened and the rib is uncovered. In the authors' initial experience, an endoscopic Kerri son rongeur was used to cut away the rib. However, from the fourth procedure, a switch was made to a surgical drill (Midas Rex, Medtronic, Inc, Dublin, Ireland) with a $7 \mathrm{~mm}$ diamond bur facilitatedtheresection a lot (Figure 3). In the authors' opinion, a more precise resectionisobtained using a drill. Also, less tissue damage is caused because the Kerri son rongeur doesn't need to be hooked in the tissues above the rib. Furthermore, a smaller sized trocar $(7,5 \mathrm{~mm})$ can be used in combination with the diamond bur.

In total $6 \mathrm{~cm}$ of the rib is resected. The resection of the rib happens thoracoscopically as no drills are currently available for the robot (Figure 4).

\section{Step 3: Rib excision and extraction}

After incising the rib anteriorly and posteriorly, the lateral border of the first rib is grasped with a large clip applier by the left robotic arm. This maneuver reveals the upside of the rib. Then, the costoclavicular 


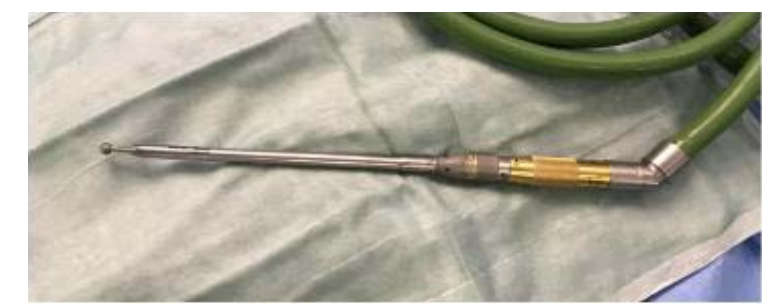

Figure 3: Close-up of the Midas Rex® surgical drill with diamond bur.

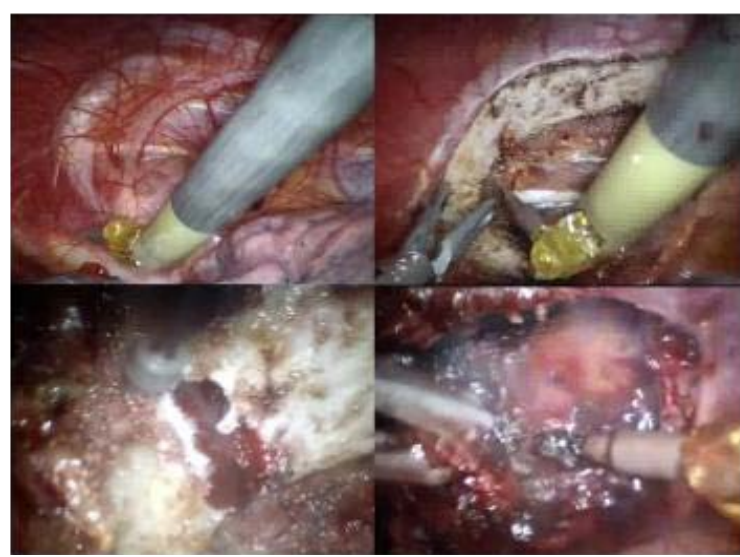

Figure 4: Perioperative images of a FRR. Upper-left corner: initial entry of the thoracic cavity. Upper-right corner: dissection of the parietal pleura exposing the first rib. Lower-left corner: incision of the rib with the surgical drill. Lowerright corner: release of the scalene muscles.

ligament, the anterior and middle scalene muscle and the border of the subclavian muscle are released with a cautery spatula, installed on the right robotic arm (Figure 4).

\section{Step 4: Rib extraction and chest tube placement}

Once all the insertions on the rib are released, the excised part is ready to be removed. This last phase is done thoracoscopically. The rib is grasped with a sponge forceps and extracted via the camera port. If the part is too big, it's further cut down with the drill. After extraction of the rib, the thoracic cavity is rinsed and checked for bleeding. If necessary, a local hemostat is applied on the surgical site.

After extraction of the rib, a small chest tube (24 Charrière) is inserted through the anterior working incision.

\section{Materials and Methods}

\section{Study design}

Asingle-center, retrospective study based on a prospective database was conducted. All patients $(\mathrm{n}=11)$ undergoing a robotassisted FFR in the treatment of TOS ( $n=11)$ from January 2017 to August 2018 (in one patient both sides were treated) were included.

Patient characteristics were collected. The operative outcomes were operative time, postoperative pain and complications, mean hospital stay and postoperative outcome (Table 1).

\section{Data analysis}

Medical records were used together with a prospective database to collect the data.
Data points were analyzed for age, gender, affected side, and type of pathology, total operative time, surgery time, pain score, morbidity, mortality and mean hospital stay.

Operative times were reported as mean \pm standard deviation.

Follow-up was performed after 6 weeks by clinical evaluation.

\section{Results}

Surgery was performed on 11 patients (12 FRR's), all except one were female. The average age of intervention was 37 . Eleven cases were diagnosed as nTOS (some of which had a combination of aTOS). One patient was treated for nTOS. The surgical times ranged from 55 to 180 minutes, with a mean of $99 \pm 32$ minutes.

No major postoperative complications such as Horner's syndrome, a winged scapula or neurovascular damage in the affected arm were reported. No postoperative blood transfusion was needed, nor was any postoperative wound infection noticed. One patient needed a chest tube replacement for a pneumothorax. One patient needed a readmission for pleurisy one week postoperative which was readily treated with NSAID's. Mortality was zero.

The maximum average pain score (based on the visual analogue scale, VAS) was 4,6 . The mean hospital stay was 3,4 days.

Clinical outcome at 6 weeks showed a complete remission of symptoms in 9 out of 12 cases. One patient mentioned a gradually decreasing fatigue/hypoesthesia in the effected arm. One patient had residual complaints of hyperesthesia in the effected forearm. One patient noticed no real improvement after the procedure (success 5. Discussion

Nowadays, a transaxillary or a supraclavicular approach, the latter when an arterial reconstruction is necessary, is most commonly used in FRR. The authors of this paper are familiar with the transaxillary approach. However, both techniques have their limitations.

Therefore, a new minimal invasive, robot-assisted technique was proposed. After this series the authors are convinced that this new approach has some clear benefits.

First, an excellent exposure of the first rib and thoracic outletis obtained which makes it possible to precisely identify where the neurovascular compression is occurring.

Secondly, as a consequence of the excellent exposure, there is less risk of damaging the neurovascular structures.

Thirdly, a broader excision of the rib is achieved with complete release of the tendons and fibro muscular band as the costovertebral and stern costal joints are better visualized.

To the authors' current knowledge, only three groups reported on robot-assisted first rib resection so far. However, they used this technique in the treatment of vTOS (Paget-Schroetter syndrome) [7-10]. Only Kocher et al. described this technique in the treatment of nTOS in 4 patients as well [12]. Did a mere technical description of the procedure without quantifying how many patients they had treated [9].

Recently published a paper in which they shared their experience with 83 patients [10]. It is hard to compare this paper with their 
Table 1: Results per patient ( ${ }^{*}$ same patient).

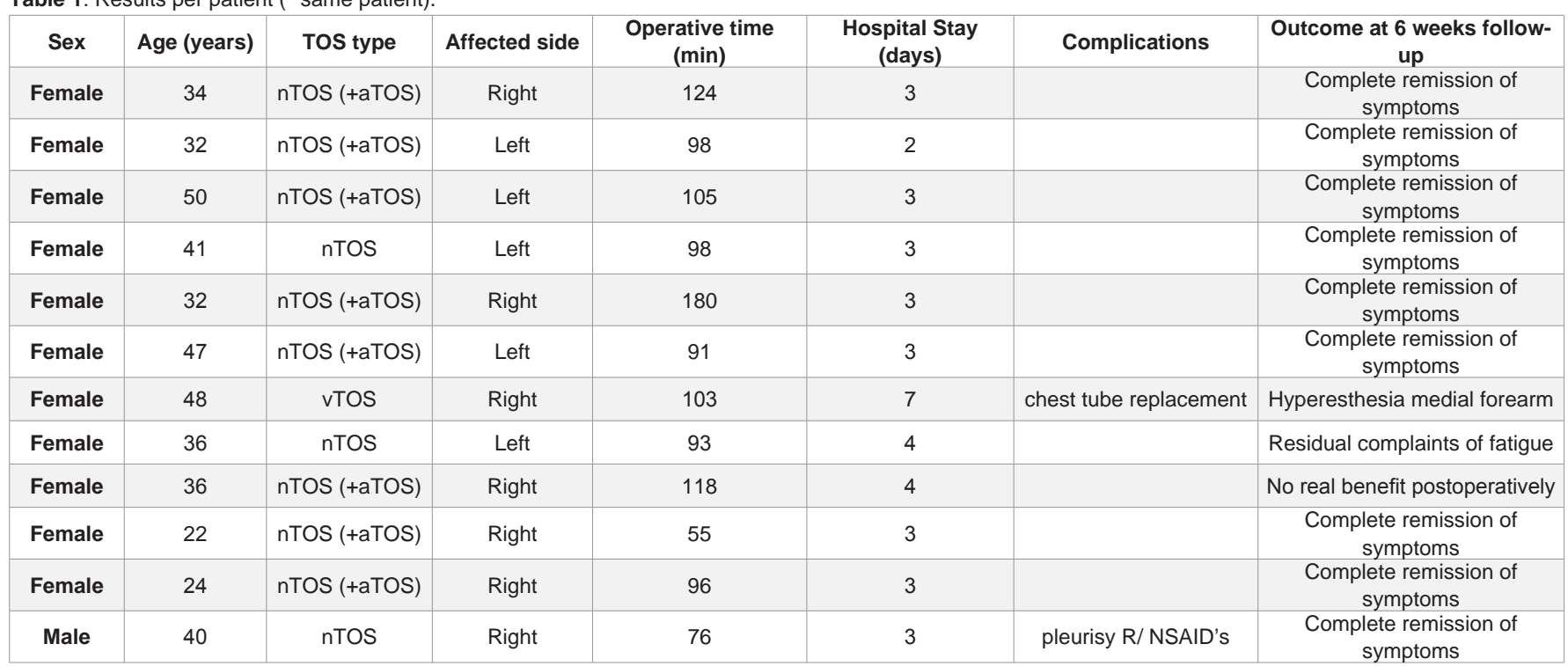

publication as they treated for vTOS. In this article, on the other hand, mainly nTOS was treated. In nTOS outcome is less binary than in vTOS. However, as the robot-assisted procedure is the same, some technical points can be compared. First, they operated in 127,6 \pm $20,8 \mathrm{~min}$, whereas operative time in this series was $99 \pm 32$ minutes. Secondly they used four trocar sites whereas only three trocars were placed by the authors as no lung retractor was used. Thirdly [13], used a Kerri son rongeur whereas a surgical drill was used in this paper. As already mentioned, the authors initially used a rongeur as well. With the drill, however, a more precise resection with less tissue damage could be obtained. Fourthly their mean hospital stay was 4 days, whereas the mean hospital stay in this article was 3,4 days.

In a very recently published article, Kocher et al. describe their experience with 8 TOS cases, 4 of which were nTOS cases. They used a Kerri son rongeur which to the authors' opinion is more dangerous than the use of a surgical drill. Also, in all but one case, an additional axillary incision was made, besides the three working ports. Through this incision a finger was placed to retract the rib. In this article a clip applier was used for this maneuver, only requiring three working ports [12].

Postoperative results were encouraging, with only one patient having no clear benefit from the procedure (i.e. success ratio of $92 \%$ ). This is comparable with the outcome of conventional techniques in large series [2,3].

\section{Conclusion}

In this paper the authors shared their experience in robot-assisted first rib resection in the treatment of nTOS. Based on the provided data, it's safe to conclude that robot-assisted first rib resection is a safe and effective way of treating TOS with minimal comorbidity. Twelve cases were treated, all but one with very encouraging results at 6 weeks follow-up. Only one patient needed readmission for pleurisy, which was pharmacologically treated.

Nowadays, open procedures (transaxillary/supraclavicular) are most commonly used. However, with a robot-assisted approach the shortcomings of these conventional techniques can be tackled. These shortcomings are: limited exposure of the operative field with the risk of damaging neurovascular structures as well as the risk of insufficient resection of the first rib. The use of the magnified three dimensionally thoracoscopic view as well as the use of robotic instrumentation bypasses these shortcomings.

Except for one recent paper, published by Kocher et al., treating 4 nTOS cases, this paper is the first to provide evidence that this technique is feasible in the treatment of nTOS.

Further research with larger series is needed to compare this new technique in a systematic way to conventional, open procedures.

\section{References}

1. Peet RM, Henriksen JD, Anderson TP, Martin GM. Thoracic-outlet syndrome: evaluation of a therapeutic exercise program. Proc Staff Meet Mayo Clin. 1956; 31: 281-287.

2. Fugate MW, Rotellini-Coltvet L, Freischlag JA. Current management of thoracic outlet syndrome. Curr Treat Options Cardiovasc Med. 2009; 11: 176-183.

3. Orlando MS, Likes KC, Mirza S, Cao Y, Cohen A, Lum YW, et al. A decade of excellent outcomes after surgical intervention in 538 patients with thoracic outlet syndrome. J Am Coll Surg. 2015; 220(5): 934-939.

4. George RS, Milton R, Chaudhuri N, Kefaloyannis E, Papagiannopoulos K. Totally Endoscopic (VATS) First Rib Resection for Thoracic Outlet Syndrome. Ann Thorac Surg. 2017; 103: 241-245.

5. Hwang J, Min BJ, Jo WM, Shin JS. Video-assisted thoracoscopic surgery for intrathoracic first rib resection in thoracic outlet syndrome. J Thorac Dis. 2017; 9: 2022-2028.

6. Nuutinen H, Riekkinen T, Aittola V, Mäkinen K, Kärkkäinen JM. Thoracoscopic Versus Transaxillary Approach to First Rib Resection in Thoracic Outlet Syndrome. Ann Thorac Surg. 2018; 105: 937-942.

7. Gharagozloo F, Meyer M, Tempesta BJ, Margolis M, Strother ET, Tummala S. Robotic en bloc first-rib resection for paget-schroetter disease, a form of thoracic outlet syndrome: Technique and initial results. Innovations (Phila). 2012; 7: 39-44

8. Neville RF, Gharagozloo F, Meyer M, Tempesta B, Tummala S. Robotic Thoracoscopic First Rib Resection and Scalenectomy for Treatment of Pagett-Schroetter Syndrome. J Vasc Surg. 2011; 54: 1854-1855. 
9. Strother E, Margolis M. Robotic First Rib Resection. Oper Tech Thorac Cardiovasc Surg. 2015; 20: 176-188.

10. Gharagozloo F, Meyer M, Tempesta B, Gruessner S. Robotic transthoracic first-rib resection for Paget-Schroetter syndrome. Eur J Cardio-Thoracic Surg [Internet]. 2018; 0: 1-6.

11. Wybaillie E, Maene L, Cooreman F, Beelen R. Robotically-Assisted Transthoracic Cervical Rib Resection. Ann Thorac Surg. 2018.
12. Kocher GJ, Zehnder A, Lutz JA, Schmidli J, Schmid RA. First Rib Resection for Thoracic Outlet Syndrome: The Robotic Approach. World J Surg. 2018; 42: $3250-3255$.

13. Intuitive I. da Vinci Surgical System®. 2018.
Austin J Surg - Volume 6 Issue 5 - 2019

ISSN : 2381-9030 | www.austinpublishing group.com

Roel et al. () All rights are reserved
Citation: Peter-Jan V, Elke W, Lieven M and Roel B. Robot-Assisted First Fib Resection for Neurogenic Thoracic Outlet Syndrome. Austin J Surg. 2019; 6(5): 1171. 